\title{
NEW GENERATING FUNCTIONS FOR MULTIVARIATE BIORTHOGONAL POLYNOMIALS ON THE N-SPHERE
}

\author{
HAROLD EXTON \\ "Nyuggel”, Lunabister, Dunrossness \\ Shetland ZE2 9JH, UNITED KINGDOM
}

(Received July, 1994; revised December, 1994)

\begin{abstract}
Certain multivariate biorthogonal polynomials on the $\mathrm{N}$-sphere arise in connection with quantum chromodynamics. These functions can be expressed in terms of Lauricella functions of the first kind, and multi-dimensional generating functions for them are deduced by means of an extension of Bailey's theorem.
\end{abstract}

Key words: Generating Functions, Biorthogonal Polynomials.

AMS subject classification: $33 \mathrm{C} 30,33 \mathrm{C} 45,81 \mathrm{~V} 05$.

\section{Introduction and Notation}

The purpose of this study is to give a number of new generating functions for the biorthogonal polynomials on the $\mathrm{N}$-sphere first presented by Lam and Tratnik [8], and discussed at considerable length by Kalnins, Miller and Tratnik [6]. These polynomials, which arose in certain calculations relating to quantum chromodynamics, may be represented as Lauricella functions $F_{A}^{(n)}$ in the form

$$
F_{A}^{(n)}\left(M+G-1,-m_{1}, \ldots,-m_{n} ; g_{1}, \ldots, g_{n} ; x_{1}, \ldots, x_{n}\right)
$$

and

$$
(1-X)^{M} F_{A}^{(n)}\left(1-M-g_{n+1},-m_{1}, \ldots,-m_{n} ; g_{1}, \ldots, g_{n} ; x_{1} /(X-1), \ldots, x_{n} /(X-1)\right)
$$

where $G=g_{1}+\ldots+g_{n+1}$.

The basis of the results given is a multi-dimensional generalization of Bailey's theorem as given by Exton [4], p. 139 which extends the discussion of Bailey [2] and Slater [9], p. 58. This very general formulation may be stated as follows:

$$
\begin{aligned}
& \text { If } B_{m_{1}}, \ldots,=\sum_{p_{1}, \ldots, p_{n}=0}^{m_{1}, \ldots, m_{n}} A_{p_{1}}, \ldots, p_{n} U_{m_{1}-p_{1}, \ldots, m_{n}-p_{n}} V_{m_{1}+p_{1}, \ldots, m_{n}+p_{n}} \text { and } \\
& C_{m_{1}, \ldots, m_{n}}=\sum_{1}=m_{1}, \ldots, p_{n}=m_{n} D_{p_{1}, \ldots, p_{n}} U_{p_{1}-m_{1}, \ldots, p_{n}-m_{n}} V_{p_{1}}+m_{1}, \ldots, p_{n}+m_{n}, \\
& \text { then, formally, } \\
& \sum A_{m_{1}, \ldots, m_{n}} C_{m_{1}, \ldots, m_{n}}=\sum B_{m_{1}, \ldots, m_{n}} D_{m_{1}, \ldots, m_{n}}
\end{aligned}
$$


It is understood that $A, U, D$ and $V$ are functions of $p_{1}, \ldots, p_{n}$ only, and any questions of convergence must be dealt with in each individual case as appropriate. The symbol $\sum$ without further qualification denotes a summation with the indices of summation running over all nonnegative integer values.

As usual, we employ the notation

$$
(a, n)=a(a+1)(a+2) \ldots(a+n-1)=\Gamma(a+n) / \Gamma(a) ; \quad(a, 0)=1,
$$

and following Tratnik [12] for example, we put

$$
x_{1}+\ldots+x_{n}=X \text { etc. }
$$

The generalization hypergeometric function of one variable is given by

$$
\begin{gathered}
A^{F} B^{\left(a_{1}, \ldots, a_{A} ; b_{1}, \ldots, b_{B} ; x\right)}=\sum \frac{\left(a_{1}, m\right) \ldots\left(a_{A}, m\right) x^{m}}{\left(b_{1}, m\right) \ldots\left(b_{B}, m\right) m !} \\
=\sum \frac{((a), m) x^{m}}{((b), m) m !}
\end{gathered}
$$

where, for convenience, the sequence of parameters $a_{1}, \ldots, a_{A}$ is denoted by $(a)$ etc. Certain multiple hypergeometric functions also figure in the analysis and are given as follows:

The Lauricella function of the first kind

$$
\begin{aligned}
& F_{A}^{(n)}\left(a, b_{1}, \ldots, b_{n} ; c_{1}, \ldots, c_{n} ; x_{1}, \ldots, x_{n}\right) \\
= & \sum \frac{(a, M)\left(b_{1}, m_{1}\right) \ldots\left(b_{n}, m_{n}\right) x_{1}^{m_{1}} \ldots x_{n}^{m_{n}}}{\left(c_{1}, m_{1}\right) \ldots\left(c_{n}, m_{n}\right) m_{1} ! \ldots m_{n} !}
\end{aligned}
$$

the Lauicella function of the third kind

$$
\begin{aligned}
& F_{C}^{(n)}\left(a, b ; c_{1}, \ldots, c_{n} ; x_{1}, \ldots, x_{n}\right) \\
= & \sum \frac{(a, M)(b, M) x_{1}^{m_{1}} \ldots x_{n}^{m_{n}}}{\left(c_{1}, m_{1}\right) \ldots\left(c_{n}, m_{n}\right) m_{1} ! \ldots m_{n} !}
\end{aligned}
$$

and Karlsson's [7] generalized Kampé de Fériet function

$$
\begin{gathered}
F_{C: D}^{A: B}\left[\begin{array}{l}
(a):\left(b_{1}\right) ; \ldots ;\left(b_{n}\right) ; \\
\left.(c):\left(d_{1}\right) ; \ldots ;\left(d_{n}\right) ; x_{1}, \ldots, x_{n}\right]
\end{array}\right. \\
=\sum \frac{((a), M)\left(\left(b_{1}\right), m_{1}\right) \ldots\left(\left(b_{n}\right), m_{n}\right) x_{1}^{m_{1}} \ldots x_{n}^{m_{n}}}{((c), M)\left(\left(d_{1}\right), m_{1}\right) \ldots\left(\left(d_{n}\right), m_{n}\right) m_{1} ! \ldots m_{n} !} .
\end{gathered}
$$

It will be seen that

and

$$
\begin{gathered}
F_{A}^{(n)}\left(a, b_{1}, \ldots, b_{n} ; c_{1}, \ldots, b_{n} ; x_{1}, \ldots, x_{n}\right) \\
=F_{0: 1}^{1: 1}\left[\begin{array}{l}
a: b_{1} ; \ldots ; b_{n} ; \\
-: c_{1} ; \ldots ; c_{n} ; x_{1}, \ldots, x_{n}
\end{array}\right] \\
F_{C}^{(n)}\left(a, b ; c_{1}, \ldots, c_{n} ; x_{1}, \ldots, x_{n}\right)
\end{gathered}
$$

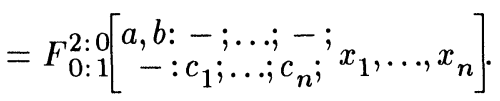


Any values of the parameters for which any of the expressions given in this paper do not make sense are tacitly excluded. For any relevant general information, the reader is referred to Appell et Kampé de Fériet [1], Erdélyi [3], Exton [4], Slater [9], Srivastava and Karlsson [10], and Srivastava and Manocha [11], for example.

\section{Main Results}

A generating relation of quite general character for the polynomial (1.1) may be deduced from (1.3). Put

and

$$
\begin{gathered}
U=1 /\left(m_{1} ! \ldots m_{n} !\right), V=(G-1, M) \\
A=\frac{\left(-x_{1}\right)^{m_{1}} \ldots\left(-x_{n}\right)^{-m_{n}}}{\left(g_{1}, m_{1}\right) \ldots\left(g_{n}, m_{n}\right) m_{1} ! \ldots m_{n} !} \\
D=\frac{((p), M)\left(\left(h_{1}\right), m_{1}\right) \ldots\left(\left(h_{n}\right), m_{n}\right) t_{1}^{m_{1}} \ldots t_{n}^{m_{n}}}{((q), M)\left(\left(k_{1}\right), m_{1}\right) \ldots\left(\left(k_{n}\right), m_{n}\right)} .
\end{gathered}
$$

After some rather tedious manipulation, we obtain the expression

$$
\begin{aligned}
& \sum \frac{((p), M)(G-1, M)\left(\left(h_{1}\right), m_{1}\right) \ldots\left(\left(h_{n}\right), m_{n}\right) t_{1}^{m_{1}} \ldots t_{n}^{m_{n}}}{((q), M)\left(\left(k_{1}\right), m_{1}\right) \ldots\left(\left(k_{n}\right), m_{n}\right) m_{1} ! \ldots m_{n} !} \\
& \cdot F_{A}^{(n)}\left(M+G-1,-m_{1}, \ldots,-m_{n} ; g_{1}, \ldots, g_{n} ; x_{1}, \ldots, x_{n}\right) \\
& =\sum \frac{((p), M)(G-1,2 M)\left(\left(h_{1}\right), m_{1}\right) \ldots\left(\left(h_{n}\right), m_{n}\right)\left(-x_{1} t_{1}\right)^{m_{1}} \ldots\left(-x_{n} t_{n}\right)^{m_{n}}}{((q), M)\left(g_{1}, m_{1}\right) \ldots\left(g_{n}, m_{n}\right)\left(\left(k_{1}\right), m_{1}\right) \ldots\left(\left(k_{n}\right), m_{n}\right) m_{1} ! \ldots m_{n} !} \\
& \cdot F_{Q: K}^{P+1: H}\left[\begin{array}{c}
(p)-M, G-1+2 M:\left(h_{1}\right)+m_{1} ; \ldots ;\left(h_{n}\right)+m_{n} ; \\
(q)+M:\left(k_{1}\right)+m_{1} ; \ldots ;\left(k_{n}\right)+m_{n} ;
\end{array}\right] .
\end{aligned}
$$

This is the required generating function for the polynomial (1.1).

Similarly, on putting

and

$$
\begin{gathered}
U=\frac{1}{\left(g_{n+1}, M\right) m_{1} ! \ldots m_{n} !}, \quad V=1, \\
A=\frac{x_{1}^{m_{1}} \ldots x_{n}^{m_{n}}(X-1)^{-M}}{\left(g_{1}, m_{1}\right) \ldots\left(g_{n}, m_{n}\right) m_{1} ! \ldots m_{n} !}
\end{gathered}
$$

nd

$$
D=\frac{((p), M)\left(\left(h_{1}\right), m_{1}\right) \ldots\left(\left(h_{n}\right), m_{n}\right)(X-1)^{M} t_{1}^{m_{1}} \ldots t_{n}^{m_{n}}}{((q), M)\left(\left(k_{1}\right), m_{1}\right) \ldots\left(\left(k_{n}\right), m_{n}\right)}
$$

we obtain a general generating function for the polynomial (1.2), namely

$$
\begin{gathered}
\sum \frac{((p), M)\left(\left(h_{1}\right), m_{1}\right) \ldots\left(\left(h_{n}\right), m_{n}\right) t_{1}^{m_{1}} \ldots t_{n}^{m_{n}}(X-1)^{M}}{((q), M)\left(\left(k_{1}\right), m_{1}\right) \ldots\left(\left(k_{n}\right), m_{n}\right) m_{1} ! \ldots m_{n} !\left(g_{n+1}, M\right)} \\
\cdot F_{A}^{(n)}\left(1-g_{n+1}-M,-m_{1}, \ldots,-m_{n} ; g_{1}, \ldots, g_{n} ; x_{1} /(X-1), \ldots, x_{n} /(X-1)\right)
\end{gathered}
$$




$$
\begin{gathered}
=\sum \frac{((p), M)\left(\left(h_{1}\right), m_{1}\right) \ldots\left(\left(h_{n}\right), m_{n}\right)\left(x_{1} t_{1}\right)^{m_{1}} \ldots\left(x_{n} t_{n}\right)^{m_{n}}}{((q), M)\left(g_{1}, m_{1}\right) \ldots\left(g_{n}, m_{n}\right)\left(\left(k_{1}\right), m_{1}\right) \ldots\left(\left(k_{n}\right), m_{n}\right) m_{1} ! \ldots m_{n} !} \\
\cdot F_{Q+1: K}^{P: H}\left[\begin{array}{c}
(p)+M:\left(h_{1}\right)+m_{1} ; \ldots ;\left(h_{n}\right)+m_{n} ; \\
(q)+M, g_{n+1}:\left(k_{1}\right)+m_{1}: \ldots:\left(k_{n}\right)+m_{n} ;
\end{array} t_{1}(X-1), \ldots, t_{n}(X-1)\right] .
\end{gathered}
$$

\section{Compact Generating Relations}

A number of more compact expressions can be obtained as special cases of (2.1) and (2.2). In the first of these, suppose that $P=Q=H=K=0$, when the inner generalized Kampé de Fériet function on the right becomes

$$
{ }_{1} F_{0}(G-1+2 M ;-; T)=(1-T)^{1-G-2 M}
$$

as a simple consequence of the binomial theorem.

Hence, we see that

$$
\begin{aligned}
& \sum \frac{(G-1, M) t_{1}^{m_{1}} \ldots t_{n}^{m_{n}}}{m_{1} ! \ldots m_{n} !} F_{A}^{(n)}\left(M+G-1, m_{1}, \ldots,-m_{n} ; g_{1}, \ldots, g_{n} ; x_{1}, \ldots, x_{n}\right) \\
= & (1-T)^{1-G_{C}} F_{C}^{(n)}\left(\frac{1}{2} G-\frac{1}{2}, \frac{1}{2} G ; g_{1}, \ldots, g_{n} ;-4 t_{1} x_{1}(1-T)^{-2}, \ldots, t_{n} x_{n}(1-T)^{-2}\right) .
\end{aligned}
$$

Next, put $t_{1}=\ldots=t_{n}=t$ and take $P=K=0$ and $H=Q=1$. After letting $q=H$, we then have

$$
\begin{gathered}
\sum \frac{(G-1, M)\left(h_{1}, m_{1}\right) \ldots\left(h_{n}, m_{n}\right) t^{M}}{(H, M) m_{1} ! . . m_{n} !} F_{A}^{(n)}\left(M+G-1,-m_{1}, \ldots,-m_{n} ; g_{1}, \ldots, g_{n} ; x_{1}, \ldots, x_{n}\right) \\
=(1-t)^{1-G} F_{1: 1}^{2: 1}\left[\begin{array}{c}
\frac{1}{2} G-\frac{1}{2}, \frac{1}{2} G: h_{1} ; \ldots ; h_{n} ; \\
H: g_{1} ; \ldots ; g_{n} ;
\end{array} m^{-2}-4 x_{1} t(1-t)^{-2}, \ldots,-4 x_{n} t(1-t)^{-2}\right] .
\end{gathered}
$$

Further, put $g_{1}=h_{1}, \ldots, g_{n}=h_{n}$ and obtain

$$
\begin{gathered}
\sum \frac{(G-1, M) t^{M}}{(H, M) m_{1} ! \ldots m_{n} !} F_{A}^{(n)}\left(M+G-1,-m_{1}, \ldots,-m_{n} ; h_{1}, \ldots, h_{n} ; x_{1}, \ldots, x_{n}\right) \\
=(1-t)^{1-G}{ }_{2} F_{1}\left(\frac{1}{2} G-\frac{1}{2}, \frac{1}{2} G ; H ;-4 t X(1-t)^{-2}\right.
\end{gathered}
$$

A general result of quite a different character may be deduced by putting

$$
t_{n}=-t_{1}-\ldots-t_{n-1}
$$

so that, if we let $H=K=0$, the inner generalized Kampé de Fériet on the right of (2.1) reduces to unity by means of the binomial theorem. Compare Exton [5]. Hence,

$$
\begin{gathered}
\sum \frac{((p), M)(G-1, M) t_{1}^{m_{1}} \ldots t_{n-1}^{m_{n}-1}\left(-t_{1}-\ldots-t_{n-1}\right)^{m_{n}}}{((q), M) m_{1} ! \ldots m_{n} !} \\
\cdot F_{A}^{(n)}\left(M+G-1,-m_{1}, \ldots,-m_{n} ; g_{1}, \ldots, g_{n} ; x_{1}, \ldots, x_{n}\right) \\
=F_{Q: 1}^{P+2: 0}\left[\begin{array}{c}
\left.(p), \frac{1}{2} G-\frac{1}{2}, \frac{1}{2} G:-; \ldots ;-;-4 x_{1} t_{1}, \ldots,-r x_{n-1} t_{n-1}, 4 x_{n}\left(t_{1}+\ldots+t_{n-1}\right)\right] . \\
(q): g_{1} ; \ldots ; g_{n} ;
\end{array}\right.
\end{gathered}
$$


The previous expansion embodies some degree of flexibility, since the $p$ and $q$ parameters can be chosen arbitrarily in order to simplify the result. For example, if $P=0$ and $Q=2$ such that $q_{1}=\frac{1}{2} G-\frac{1}{2}$ and $q_{2}=\frac{1}{2} G$, the right-hand member of (3.1) reduces to the product of several ${ }_{0} F_{1}$ series, which are, effectively, Bessel functions. We then see that

$$
\begin{gathered}
\sum \frac{(G-1, M) t_{1}^{m_{1}} \ldots t_{n-1}^{m_{n}-1}\left(-t_{1}-\ldots-t_{n-1}\right)^{m_{n}}}{\left(\frac{1}{2} G-\frac{1}{2}, M\right)\left(\frac{1}{2} G, M\right) m_{1} ! \ldots m_{n} !} \\
\cdot F_{A}^{(n)}\left(M+G-1,-m_{1}, \ldots,-m_{n} ; g_{1}, \ldots, g_{n} ; x_{1}, \ldots, x_{n}\right) \\
={ }_{0} F_{1}\left(-; g_{1} ;-4 x_{1} t_{1}\right) \ldots{ }_{0} F_{1}\left(-; g_{n-1} ;-4 x_{n-1} t_{n-1}\right)_{0} F_{1}\left(-; g_{n} ; 4 x_{n}\left(t_{1}+\ldots+t_{n-1}\right)\right) .
\end{gathered}
$$

The second general result, (2.2), may also be used to obtain a number of expansion. Suppose that $P=H=Q=K=0$, when the inner generalized Kampé de Fériet function on the right takes the form

$$
{ }_{0} F_{1}\left(-; g_{n+1} ; T(X-1)\right)
$$

and we have the generating function

$$
\begin{gathered}
\sum \frac{t_{1}^{m_{1}} \ldots t_{n}^{m_{n}}(X-1)^{M}}{\left(g_{n+1}, M\right) m_{1} ! \ldots m_{n} !} \\
\cdot F_{A}^{(n)}\left(1-M-g_{n+1},-m_{1}, \ldots,-m_{n} ; g_{1}, \ldots, g_{n} ; x_{1} /(X-1), \ldots, x_{n} /(X-1)\right)
\end{gathered}
$$

Put $H=1, K=0$ and $t_{1}=\ldots=t_{n}=t$, and obtain the result

$$
\begin{gathered}
\sum \frac{\left((p, M)\left(h_{1}, m_{1}\right) \ldots\left(h_{n}, m_{n}\right) t^{M}(X-1)^{M}\right.}{((q), M)\left(g_{n+1}, M\right) m_{1} ! \ldots m_{n} !} \\
\cdot F_{A}^{(n)}\left(1-M-g_{n+1},-m_{1}, \ldots,-m_{n} ; g_{1}, \ldots, g_{n} ; x_{1} /(X-1), \ldots, x_{n} /(X-1)\right) \\
=\sum \frac{((p), M)\left(h_{1}, m_{1}\right) \ldots\left(h_{n}, m_{n}\right) x_{1}^{m_{1}} \ldots x_{n}^{m_{n}} n t^{M}}{((q), M)\left(g_{1}, m_{1}\right) \ldots\left(g_{n}, m_{n}\right) m_{1} ! \ldots m_{n} !} \\
\cdot{ }_{P+1} F_{Q+1}\left((P)+M, H+M ;(q)+M, g_{n+1} ; t(X-1)\right) .
\end{gathered}
$$

Further, let $P=0, Q=1$ and $q=h$, when it is found that

$$
\begin{gathered}
\sum \frac{\left(h_{1}, m_{1}\right) \ldots\left(h_{n}, m_{n}\right) t^{M}(X-1)^{M}}{(H, M)\left(g_{n+1}, M\right) m_{1} ! \ldots m_{n} !} \\
\cdot F_{A}^{(n)}\left(1-M-g_{n+1}, m_{1}, \ldots,-m_{n} ; g_{1}, \ldots, g_{n} ; x_{1} /(X-1), \ldots, x_{n} /(X-1)\right) \\
={ }_{0} F_{1}\left(-; g_{1} ; x_{1} t\right) \ldots{ }_{0} F_{1}\left(-; g_{n} ; x_{n} t\right)_{0} F_{1}\left(-; g_{n+1} ; t(X-1)\right) .
\end{gathered}
$$

In (2.2), let $H=K=0$ and $t_{n}=-t_{1}-\ldots-t_{n-1}$, when, as in (3.1), the inner generalized Kampé de Fériet function reduces to unity, so that

$$
\begin{gathered}
\sum \frac{((p), M) t_{1}^{m_{1}} \ldots t_{n-1}^{m_{n}-1}\left(-t_{1}-\ldots-t_{n-1}\right)^{m} n(X-1)^{M}}{((q), M)\left(g_{n+1}, M\right) m_{1} ! \ldots m_{n} !} \\
\cdot F_{A}^{(n)}\left(1-M-g_{n+1},-m_{1}, \ldots,-m_{n} ; g_{1}, \ldots, g_{n} ; x_{1} /(X-1), \ldots, x_{n} /(X-1)\right)
\end{gathered}
$$




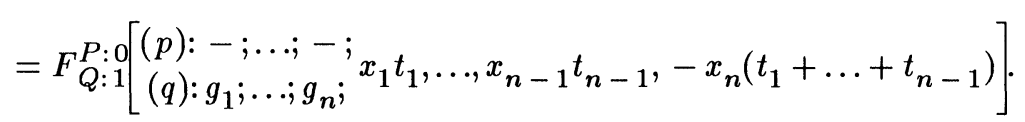

Put $P=Q=0$, and obtain the result

$$
\begin{gathered}
\sum \frac{t_{1}^{m_{1}} \ldots t_{n-1}^{m_{n}-1}\left(-t_{1}-\ldots-t_{n-1}\right)^{m_{n}}(X-1)^{M}}{((q), M)\left(t_{n+1}, M\right) m_{1} ! \ldots m_{n} !} \\
\cdot F_{A}^{(n)}\left(1-M-g_{n+1},-m_{1}, \ldots,-m_{n} ; g_{1}, \ldots, g_{n} ; x_{1} /(X-1), \ldots, x_{n} /(X-1)\right) \\
={ }_{0} F_{1}\left(-; g_{1} ; x_{1} t_{1}\right) \ldots{ }_{0} F_{1}\left(-; g_{n-1} ; x_{n-1} t_{n-1}\right)_{0} F_{1}\left(-; g_{n} ;-x_{n}\left(t_{1}+\ldots+t_{n-1}\right)\right) .
\end{gathered}
$$

The formulas (3.2) and (3.3) again may be expressed in terms of Bessel functions on account of the occurrence of the ${ }_{0} F_{1}$ series on the right in each case.

\section{References}

[1] Appell, P. and Kampé de Fériet, J., Fonctions Hypergéometriques et Hypersphériques, Gauthier Villars, Paris 1926.

[2] Bailey, W.N., Identities of Rogers-Ramanujan type, Proc. London Math. Soc. 50 (1949), 110.

[3] Erdélyi, A., Higher Transcendental Functions, McGraw Hill, New York 1953.

[4] Exton, H., Multiple Hypergeometric Functions, Ellis Horwood, Chicester, UK 1976.

[5] Exton, H., Generating relations for Tratnik's multivariate biorthogonal continuous Hahn polynomials, J. Math. Phys. 33 (1992), 524-527.

[6] Kalnins, E.G., Miller, W. and Tratnik, M.V., Families of orthogonal and biorthogonal polynomials on the N-sphere, SIAM J. Math. Anal. 22 (1991), 272-294.

[7] Karlsson, P.W., Reduction of certain generalized Kampé de Fériet functions, Math. Scand. 32 (1973), 265-268.

[8] Lam, C.S. and Tratnik, M.V., Conformally invariant operator-product expansions of any number of operators of arbitrary spin, Canad. J. Phys. 63 (1985), 1427-1437.

[9] Slater, L.J., Generalized Hypergeometric Functions, Cambridge University Press 1966.

[10] Srivastava, H.M. and Karlsson, P.W., Multiple Gaussian Hypergeometric Series, Ellis Horwood, Chichester, UK 1985.

[11] Srivastava, H.M. and Manocha, H.L., A Treatise on Generating Functions, Ellis Horwood, Chichester, UK 1984.

[12] Tratnik, M.V., Multivariate biorthogonal Hahn polynomials, J. Math. Phys. 30 (1989), 627-634. 


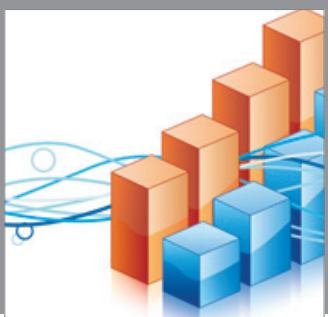

Advances in

Operations Research

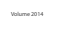

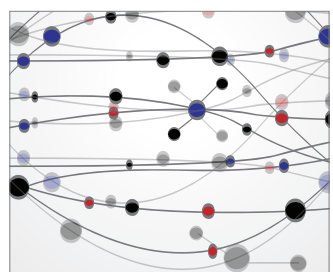

\section{The Scientific} World Journal
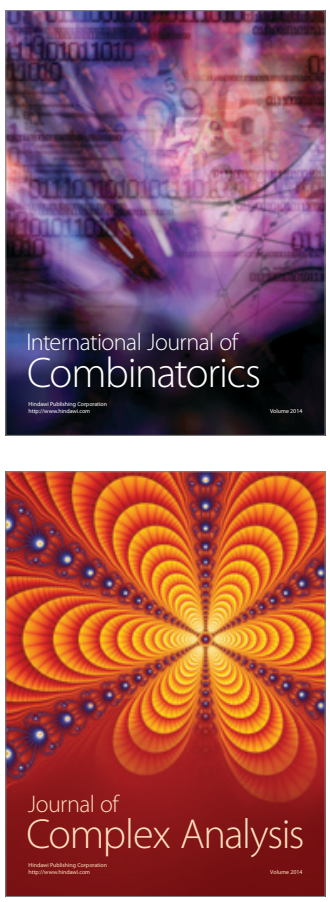

International Journal of

Mathematics and

Mathematical

Sciences
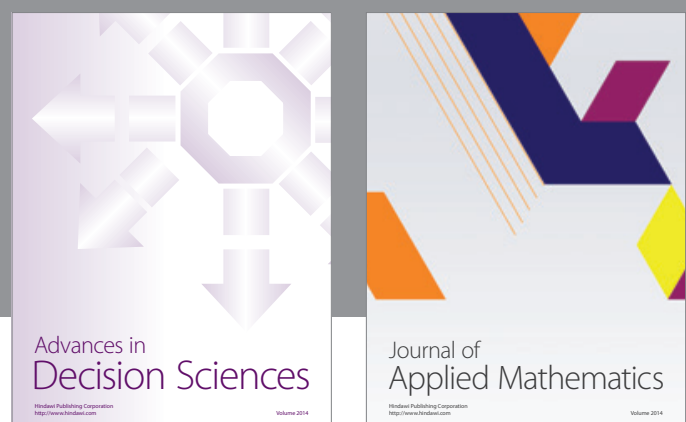

Journal of

Applied Mathematics
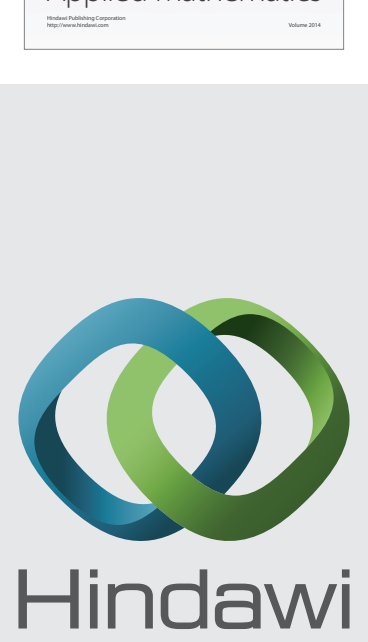

Submit your manuscripts at http://www.hindawi.com
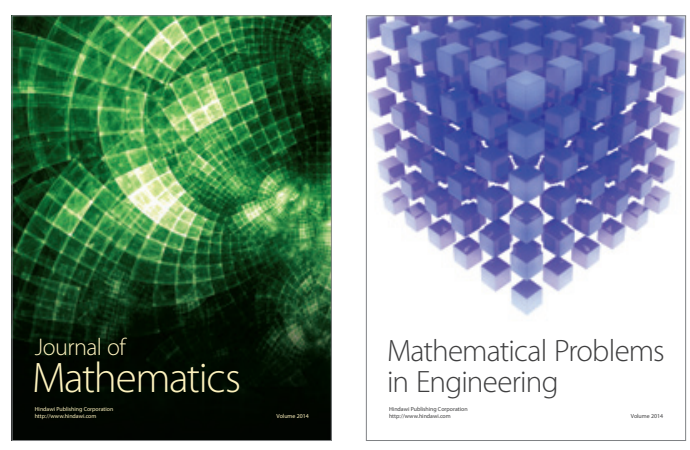

Mathematical Problems in Engineering
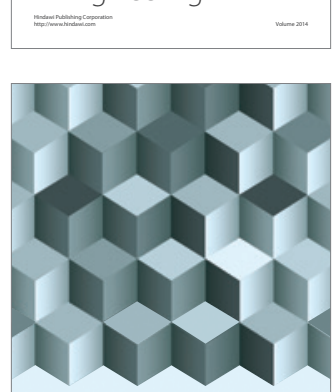

Journal of

Function Spaces
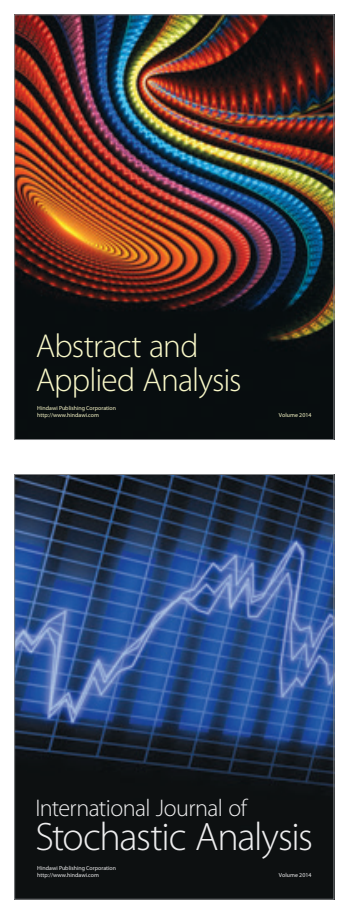

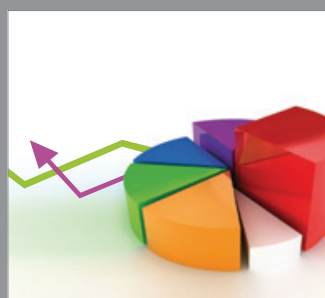

ournal of

Probability and Statistics

Promensencen
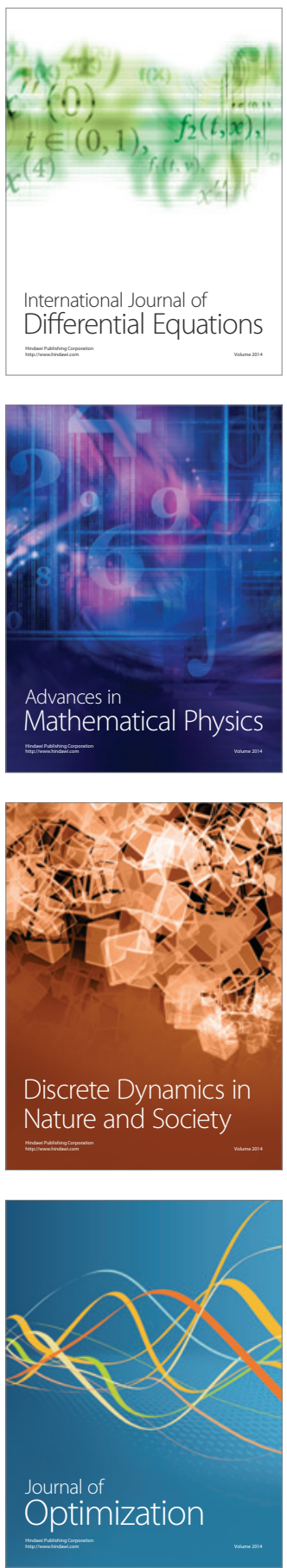